\title{
ЧАСТОТА НАРУШЕНИЙ УГЛЕВОДНОГО ОБМЕНА У ПАЦИЕНТОВ ДНЕВНОГО СТАЦИОНАРА С ПОГРАНИЧНЫМИ ЗНАЧЕНИЯМИ ГЛИКЕМИИ НАТОЩАК И ХОТЯ БЫ ОДНИМ ФАКТОРОМ РИСКА РАЗВИТИЯ САХАРНОГО ДИАБЕТА
}

\author{
В. В. Боева ${ }^{\natural}$, Т. А. Боева ${ }^{2}$ А. Н. Завьялов ${ }^{3}$
}

Федеральный клинический центр высоких медицинских технологий Федерального медико-биологического агентства России, Московская область, городской округ Химки, микрорайон Новогорск, Россия

2 Тамбовское областное государственное бюджетное учреждение здравоохранения «Тамбовская Центральная районная больница»,

Тамбовская область, Тамбовский район, с. Покрово-Пригородное, Россия

Р Российский национальный исследовательский медицинский университет имени Н. И. Пирогова, Москва, Россия

\begin{abstract}
С целью оценки диагностики нарушений углеводного обмена проведено обследование пациентов, поступивших в дневной стационар Тамбовского областного государственного бюджетного учреждения здравоохранения (ТОГ БУЗ) «Тамбовская Центральная районная больница» в период 6 месяцев 2018 г. Проведенное в рамках дневного стационара обследование 91 человека позволило выявить у 31 (34,0\%) человека сахарный диабет 2-го типа (СД), у 6 (6,5\%) нарушенную гликемию натощак и у 22 (24,1\%) нарушенную толерантность к глюкозе. Данное обследование показало необходимость расширения популяции скрининга в группах риска по развитию сахарного диабета 2 типа. Дано обоснование проведения перорального теста толерантности с 75 г глюкозы всем лицам с глюкозой венозной плазмы натощак $\geq 5,6 \leq 6,0$ ммоль/л, имеющим один или более факторы риска развития СД 2 типа и/или метаболический синдром: среди данной категории СД был выявлен в 4,3\%, а предиабет — в 14,4\% случаев.
\end{abstract}

Ключевые слова: сахарный диабет 2 типа, нарушенная гликемия натощак, нарушенная толерантность К глюкозе, скрининг, глюкоза венозной плазмы натощак, предиабет

Информация о вкладе авторов: В. В. Боева - анализ литературы, планирование исследования, анализ и интерпретация данных; Т. А. Боева — анализ литературы, планирование исследования, анализ и сбор данных; А. Н. Завьялов - анализ литературы, анализ и интерпретация данных.

Соблюдение этических стандартов: этический комитет ФГБОУ ВО РНИМУ им. Н. И. Пирогова Минздрава России; №176 от 25 июня 2018 г.

$\triangle$ Для корреспонденции: Валентина Владимировна Боева

ул. Ивановская, д. 3, Новогорск, Московская обл., 141435; BoevaVV@yandex.ru

Статья получена: 20.01.2019 Статья принята к печати: 12.03.2019 Опубликована онлайн: 15.03.2019

DOI: $10.24075 /$ vrgmu.2019.014

\section{FREQUENCY OF CARBOHYDRATE METABOLISM DISORDERS IN DAY-CARE PATIENTS WITH BORDERLINE FASTING BLOOD SUGAR LEVELS AND AT LEAST ONE RISK FACTOR FOR DIABETES MELLITUS}

Boeva $\mathrm{W}^{1} \bowtie$, Boeva $\mathrm{TA}^{2}$, Zavyalov $\mathrm{AN}^{3}$

${ }^{1}$ Federal Clinical Centre of High Medical Technologies, Moscow Region, Novogorsk, Russia

2 Tambov Central Regional Hospital,

Pokrovo Prigorodnoe Rural Settlement, Russia

${ }^{3}$ Pirogov Russian National Research Medical University, Moscow, Russia

In order to assess the diagnosis of carbohydrate metabolism disorders, day care patients from Tambov central regional hospital were investigated. The study was conducted during 6 months in 2018. The study included 91 patients and allowed the diagnosis of type 2 diabetes mellitus (DM) in 31 (34.0\%) cases, 6 (6.5\%) impaired fasting glucose and $22(24.1 \%)$ impaired glucose tolerance. This survey highlighted the necessity to expand the screening populations at risk for developing type 2 diabetes. The rational for the 75-gram oral glucose tolerance test for all individuals with fasting plasma glucose $\geq 5.6 \leq 6.0 \mathrm{mmol} / \mathrm{l}$ and having one or more risk factors for developing type 2 diabetes and / or metabolic syndrome is shown. Among these categories diabetes was detected in $4.3 \%$, and prediabetes in $14.4 \%$ of cases.

Keywords: diabetes mellitus type 2, impaired fasting glucose, impaired glucose tolerance, screening, fasting venous plasma glucose, prediabetes

Author contribution: all authors participated in conceiving and planning the study, collected and analyzing the data. Boeva W prepared the study for publication. Compliance with ethical standards: the study was approved by the Ethics Committee of Pirogov Russian Medical Research Medical University (Protocol 176 dated June 25, 2018). All patients gave informed consent to participate.

Correspondence should be addressed: Valentina V. Boeva

Ivanovskaya 3, Novogorsk, Moscow region, 141435; BoevaWV@yandex.ru

Received: 20.01.2019 Accepted: 12.03.2019 Published online: 15.03.2019

DOI: $10.24075 / \mathrm{brsmu} .2019 .014$

Сахарный диабет (СД) является важнейшей медикосоциальной проблемой. Число больных СД 2-го типа в мире к концу 2017 г. достигло 425 млн человек, а к 2045 г. эта цифра возрастет до 629 млн человек. Около 212,4 млн жителей планеты имеют недиагностированный СД 2-го типа [1]. По данным Государственного регистра СД, число больных в РФ на январь 2018 г. составило около 4,5 млн человек, среди которых у 4,1 млн был диагностирован СД 2-го типа [2]. Экстраполяция результатов исследования
NATION на всех жителей РФ позволяет предполагать, что около 20,7 млн человек имеют предиабет, а еще 4,2 млн - недиагностированный СД 2-го типа. Таким образом, реальная численность пациентов с СД 2-го типа в РФ составляет не менее 8 млн человек (около 5,5\% населения), что вместе с данными о предиабете (около 19,3\% населения) представляет значительную угрозу для общественного здоровья и здравоохранения, поскольку не менее 50\% остается недиагностированными, а 
следовательно, не получают лечения и имеют высокий риск развития осложнений из-за несвоевременной диагностики [3, 4].

Ежегодное увеличение численности больных СД в среднем по России в период с 2007 по 2012 г. составляло 173640 человек в год, ежегодный прирост - 6,23\% [5].

Согласно данным Федерального регистра сахарного диабета на 17 января 2018 г. распространенность СД 2-го типа в Тамбовской области составляет 4044,3 на 100000 человек, занимая второе место по данному показателю среди 85 субъектов РФ [2]. Это позволяет рассматривать Тамбовскую область в качестве одной из возможных пилотных площадок для углубленного изучения тенденций в эпидемиологии СД 2-го типа в России. Анализ распространенности СД 2-го типа среди взрослого населения Тамбовского района продемонстрировал увеличение этого показателя за период с 2011 по 2018 г. с 1600,6 до 2477,0 на 100000 человек. Возрастание распространенности СД 2-го типа в области, в первую очередь, связано с увеличением первичной заболеваемости СД 2-го типа с 208,1 до 242,6 [6].

Очевидно, что проблема профилактики и своевременного выявления СД 2-го типа приобретает общегосударственные масштабы, так как распространенность патологии остается достаточно высокой и демонстрирует тенденцию к дальнейшему росту, а проведение скрининга в группах высокого риска на современном этапе недостаточно эффективно. Соответственно, необходим системный популяционный подход, для обеспечения которого требуется расширение программы скрининга и профилактики на федеральном и региональном уровнях.

С 2007 по 2012 г. в РФ действовала подпрограмма «Сахарный диабет» Федеральной целевой программы «Предупреждение и борьба с социально значимыми заболеваниями». Ее целевыми индикаторами были выбраны продолжительность жизни больных и доля осложнений при СД, т. е. упор был сделан на вторичную просилактику. Федеральная программа первичной профилактики СД 2-го типа, а значит предотвращения прогрессии предиабета в диабет, отсутствует.

Термин «предиабет» был впервые предложен Всемирной организацией здравоохранения (ВОЗ) в 1965 г. С 1999 г. данный термин или эквивалентный ему 一 «ранние нарушения углеводного обмена» - предлагается использовать для общего названия двух предшествующих СД состояний нарушенной гликемии натощак $(\mathrm{HГH})$, нарушенной толерантности к глюкозе (НТГ) и их сочетания, чтобы акцентировать внимание и подчеркнуть высокий риск развития СД 2-го типа в дальнейшем (примерно 4-9\% случаев в год) [3, 7].

Тот факт, что не менее 50\% пациентов с СД 2-го типа не знают о наличии у них заболевания, может свидетельствовать о недостаточной чувствительности существующих критериев отбора пациентов для скрининга.

Распространенность метаболического синдрома (MC) в общей популяции составляет от 14 до 24\% [8]. Среди трудоспособного населения экономически развитых стран симптомокомплекс диагностируют у 20-50\% населения [9]. В России MC у лиц в возрасте старше 30 лет диагностируют в 30\% случаев, при этом количество больных непрерывно растет [10]. В 90\% случаев СД 2-го типа сочетается с абдоминальным ожирением и является клиническим исходом МС.

Таким образом, проблема своевременной диагностики СД 2-го типа представляется чрезвычайно актуальной, поскольку до сих пор, несмотря на увеличение доступности методов диагностики, СД 2-го типа часто выявляется лишь на этапе развития осложнений, т. е. через несколько лет от реального дебюта заболевания. Своевременная диагностика нарушений углеводного обмена в перспективе может снизить распространенность СД 2-го типа, развитие осложнений, инвалидизацию пациентов и, как следствие,

Исследование ГВПН

$n=91$

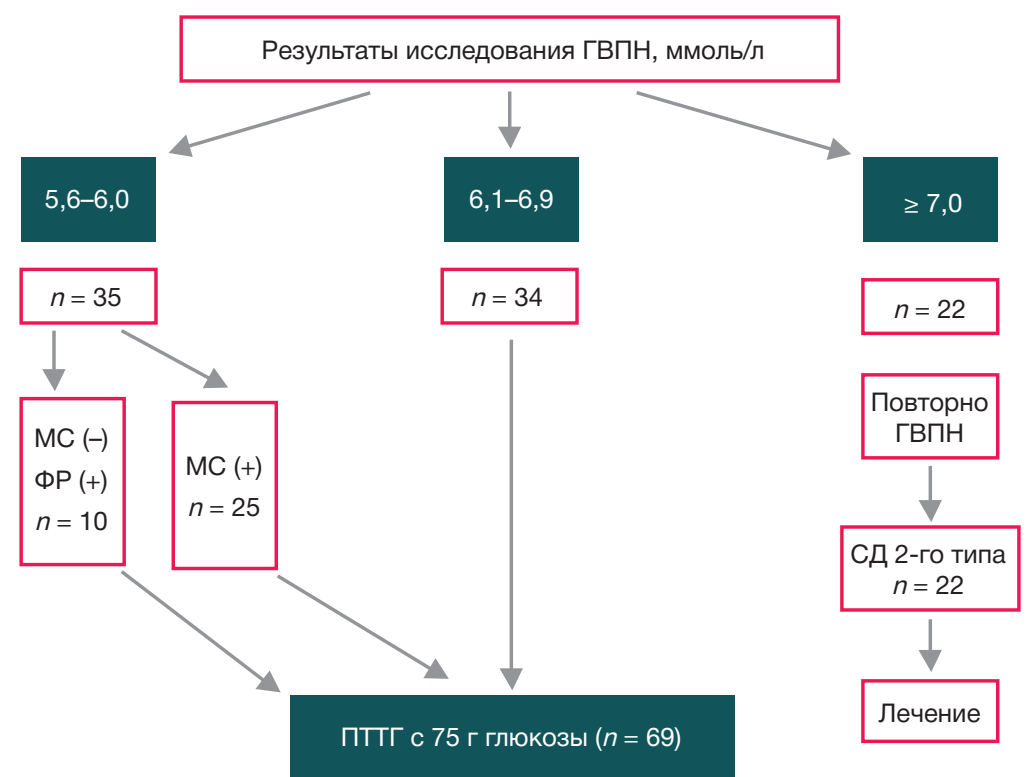

Рис. 1. В исследование были включены 91 пациент, которые были поделены на три группы в зависимости от уровня гликемии. Пациентам с двукратным повышением уровня ГВПН $\geq 7,0$ ммоль/л был выставлен диагноз СД 2-го типа и назначена терапия заболевания. Пациентам с уровнем ГВПН 6,1-6,9 ммоль/л был проведен пероральный тест толерантности к глюкозе (ПТГ). Пациентам с уровнем ГВПН 5,6-6,0 ммоль/л, имеющим факторы риска развития СД 2-го типа и метаболический синдром, также проведен ПТТГ. ГВПН — глюкоза венозной плазмы натощак 
снизить финансовую нагрузку на государственную систему здравоохранения, что является одним из приоритетных направлений ее развития. Поэтому дальнейшие исследования в данной области представляются актуальными.

\section{МАТЕРИАЛЫ И МЕТОДЫ}

Оценка методов диагностики нарушений углеводного обмена проведена у 840 пациентов, поступивших в дневной стационар ТОГ БУЗ «Тамбовская Центральная районная больница» в период 6 месяцев 2018 г. В анализ были включены 91 человек (24 мужчины и 67 женщин в возрасте от 32 до 79 лет), удовлетворяющие следующим критериям:

1) Значение глюкозы венозной плазмы натощак $(Г В П Н)$ не менее 5,6 ммоль/л.

2) Наличие одного или более факторов риска (ФР) развития СД 2-го типа [1]:

- возраст более 45 лет;

- избыточная масса тела или ожирение (ИМТ $\geq 25$ кг/M²);

- абдоминальное ожирение (окружность талии у мужчин > 94 см и у женщин > 80 см);

- артериальная гипертензия (АД $\geq 140 / 90$ мм рт. ст. или медикаментозная антигипертензивная терапия);

- гиперхолестеринемия (ЛПНП 2 1,8 ммоль/л);

- наличие сердечно-сосудистых заболеваний;

- нарушенная гликемия натощак или нарушенная толерантность к глюкозе в анамнезе;

- отягощенная наследственность по СД.

Данное обследование было одномоментным неинтервенционным когортным наблюдением. Все пациенты подписали добровольное информированное согласие до начала всех процедур.

Критерии исключения: СД 2-го типа, СД 1-го типа; обострение хронического заболевания, воспалительный процесс, тяжелая сопутствующая патология; ГВПН <5,6 ммоль/л.

Одновременно с оценкой факторов риска развития СД 2-го типа, определяли признаки метаболического синдрома. Согласно определению IDF (2005 г.), основными клиническими проявлениями метаболического синдрома у лиц европеоидной расы являются центральное ожирение (окружность талии > 94 см у мужчин и > 80 см у женщин) и два или более из следующих четырех факторов [11]:

1) повышение триглицеридов > 1,7 ммоль/л (или гиполипидемическая терапия);

2) снижение холестерина липопротеидов высокой плотности < 1,03 ммоль/л у мужчин и < 1,29 ммоль/л у женщин (или гиполипидемическая терапия);

3) повышение артериального давления (АД) $\geq 130 / 85$ мм рт. ст. (или антигипертензивная терапия);

4) содержание глюкозы в крови натощак $\geq 5,6$ ммоль/л или диагностированный ранее СД 2-го типа.

Для уточнения состояния углеводного обмена всем пациентам с уровнем ГВПН 5,6-6,0, метаболическим синдромом или хотя бы одним фактором риска СД 2-го типа, был проведен пероральный тест толерантности к глюкозе (ПТТГ) с 75 г глюкозы. Схема исследования представлена на рис. 1.

Статистическую обработку материалов работы проводили с использованием программы Statistica ver 6,1 (StatSoft; Россия) на персональном компьютере. В связи с тем, что полученные данные не подчинялись закону нормального распределения (анализ проведен методом КолмогороваСмирнова), использовали методы непараметрической статистики, критерий Манна-Уитни.

\section{РЕЗУЛЬТАТЫ ИССЛЕДОВАНИЯ}

Из 91 обследованных нами пациента 22 человека (24,2\%) самостоятельно обратились в лечебно-профилактическое учреждение (ЛПУ) в связи с впервые возникшими жалобами на повышенную утомляемость, жажду, сухость во рту, учащенное мочеиспускание, зуд кожных покровов, прибавку веса или нестабильность АД. Диагноз СД 2-го типа был подтвержден у всех 22 человек: 4 мужчин и 18 женщин в возрасте 34-69 лет, у которых при двукратном исследовании уровень ГВПН оказался $\geq 7,0$ ммоль/л.

У 35 пациентов $(38,4 \%)$ уровень ГВПН составил 5,6-6,0, выявлены метаболический синдром и один и более факторов риска СД 2-го типа. У 34 человек $(37,3 \%)$ уровень ГВПН оказался в пределах 6,1-6,9 ммоль/л, у 33 из 34 (97\%) выявлена картина метаболического синдрома. Всем пациентам данных групп был проведен ПТТГ.

Частота нарушений углеводного обмена у пациентов дневного стационара с пограничным значением гликемии натощак и хотя бы одним фактором риска СД 2-го типа составила 64,8\% ( $n=59)$, из них впервые выявленный СД 2-го типа наблюдался в 34,0\% ( $n=31)$, а предиабет в $30,7 \%(n=28)$ случаев. У лиц с предиабетом преобладала нарушенная толерантность к глюкозе - 24,1\% (22 человека).

У всех пациентов с впервые выявленным СД 2-го типа (n = 31) был обнаружен метаболический синдром, при этом у 9 человек СД 2-го типа был диагностирован по результатам ПТТГ.

Распределение пациентов в зависимости от степени нарушения углеводного обмена по результатам ПТГ в группе 35 человек с исходным значением ГВПН 5,6-6,0 ммоль/л представлено на рис. 3.

Распределение пациентов в зависимости от степени нарушения углеводного обмена по результатам ПТТГ в группе из 34 человек с исходным значением ГВПН 6,1-6,9 ммоль/л представлено на рис. 4.

Клинико-демографическая характеристика пациентов в зависимости от степени нарушений углеводного обмена ( $n=91)$ представлена в табл.1.

По данным ПТТГ ( $n=69)$, частота встречаемости СД 2-го типа составила 13,0\% (9 человек), НТГ была выявлена у 31,8\% (22 человек), НГН - у 8,7\% (6 человек), нормальная толерантность к глюкозе встречалась в 46,3\%

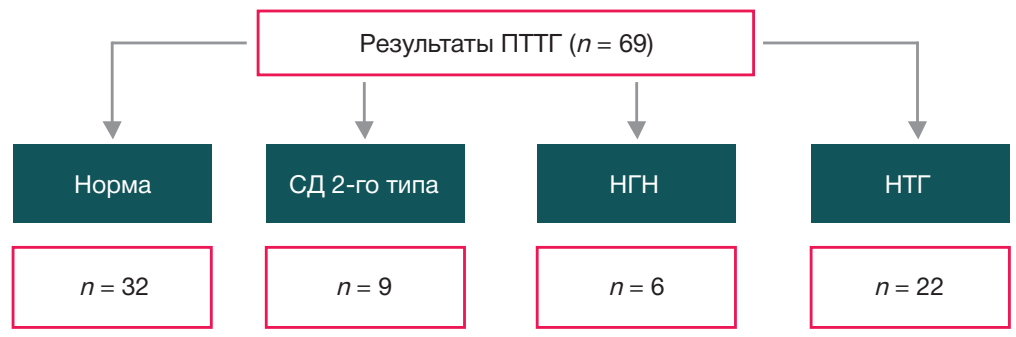

Рис. 2. Распределение пациентов в зависимости от степени нарушения углеводного обмена по результатам ПТТГ. НГН — нарушенная гликемия натощак; НТГ — нарушенная толерантность к глюкозе; ПтТГ — пероральный тест толерантности к глюкозе 
(32 человека) случаев (рис. 2). Обращает на себя внимание более низкая частота встречаемости НГН по данным ПТТГ.

Из 9 пациентов с впервые выявленным СД 2-го типа по результатам ПТТГ у 3 человек исходный уровень ГВПН соответствовал диапазону 5,6-6,0 ммоль/л и у 6 пациентов - 6,1-6,9 ммоль/л.

Распределение пациентов в зависимости от степени нарушения углеводного обмена по результатам ПТТГ в группах с исходным значением гликемии натощак равным 5,6-6,0 ммоль/л $(n=35)$ и 6,1-6,9 ммоль/л $(n=34)$, представлено в табл. 2.

Согласно полученным данным, большую часть пациентов составляли лица старше 45 лет (в 100\% случаев в группе НГН и НТГ; в 84,3\% и 90,3\% - в группах с нормальным углеводным обменом и впервые выявленным СД 2-го типа соответственно). Во всех группах преобладали пациенты с избыточной массой тела или ожирением, а также все пациенты имели увеличенное значение ОТ. Не выявлено достоверных различий по количеству пациентов с отягощенной наследственностью по СД 2-го типа (табл. 1).

Выявлено достоверно большее количество пациентов с артериальной гипертензией в группе с нормальным углеводным обменом и предиабетом в сравнении с группой с впервые выявленным СД 2-го типа $(p<0,05)$.
Достоверных различий по показателям липидного спектра, наличию сопутствующего хронического панкреатита и перенесенных сердечно-сосудистых заболеваний в группах не выявлено ( $p>0,05)$.

Следует отметить, что большее количество пациентов, имеющих в анамнезе гипергликемию, отмечено в группе впервые выявленного СД ( $p>0,05)$. Причем из 8 человек только у двоих ранее было верифицировано нарушение углеводного обмена и диагностирована нарушенная толерантность к глюкозе. Данные пациенты принимали метсормин (не более года) с последующей самостоятельной отменой. Контрольных диагностических тестов с целью уточнения состояния углеводного обмена у остальных пациентов не проводили.

Среди 4 пациентов с НТГ, у которых гипергликемию выявляли в период с 2013 по 2018 г., одна женщина непрерывно принимала метформин в дозе 500 мг в сутки в течение 6 лет. Данное обстоятельство позволило ей сохранить состояние углеводного обмена на стадии предиабета. Ни в одном случае ПТТГ с 75 г глюкозы не был проведен ранее с целью уточнения характера выявленных нарушений углеводного обмена.

У двоих пациентов с НГН в течение последнего года также был выявлен повышенный уровень ГВПН (до 6,5 ммоль/л)

Таблица 1. Сравнительная клинико-демографическая характеристика пациентов

\begin{tabular}{|l|c|c|c|c|}
\hline \multicolumn{1}{|c|}{ Оцениваемые параметры } & $\begin{array}{c}\text { Норма } \\
(n=32)\end{array}$ & $\begin{array}{c}\text { HГH } \\
(n=6)\end{array}$ & $\begin{array}{c}\text { HTГ } \\
(n=22)\end{array}$ & $\begin{array}{c}\text { СД 2-го типа } \\
(n=31)\end{array}$ \\
\hline Мужчины & $10(31,2 \%)$ & $3(50,0 \%)$ & $5(22,7 \%)$ & $6(19,3 \%)$ \\
\hline Женщины & $22(68,7 \%)$ & $3(50,0 \%)$ & $17(77,2 \%)$ & $25(80,6 \%)$ \\
\hline Возраст $\geq 45$ лет & $27(84,3 \%)$ & $6(100 \%)$ & $22(100 \%)$ & $28(90,3 \%)$ \\
\hline Отягощенная наследственность по СД 2-го типа & $2(6,2 \%)$ & $1(16,6 \%)$ & $6(27,2 \%)$ & $7(22,5 \%)$ \\
\hline ИМТ $\geq 25$ & $30(93,7 \%)$ & $6(100 \%)$ & $21(95,4 \%)$ & $31(100 \%)$ \\
\hline ОТ > 80 см (Ж) & $22(100 \%)$ & $3(100 \%)$ & $17(100 \%)$ & $25(100 \%)$ \\
\hline ОТ > 94 см (М) & $10(100 \%)$ & $3(100 \%)$ & $5(100 \%)$ & $6(100 \%)$ \\
\hline Артериальная гипертензия & $32(100 \%)^{*}$ & $6(100 \%)$ & $22(100 \%)^{*}$ & $25(80,6 \%)$ \\
\hline Гиперхолестеринемия & $21(65,6 \%)$ & $6(100 \%)$ & $15(68,1 \%)$ & $18(58,0 \%)$ \\
\hline Хронический панкреатит & $3(9,3 \%)$ & $1(16,6 \%)$ & $3(13,6 \%)$ & $5(16,1 \%)$ \\
\hline Наличие сердечно-сосудистых заболеваний: & $9(28,1 \%)$ & $2(33,3 \%)$ & $4(18,1 \%)$ & $8(25,8 \%)$ \\
\hline ОИМ & $3(9,3 \%)$ & $1(16,6 \%)$ & $2(9,0 \%)$ & $3(9,6 \%)$ \\
\hline ОНМК & $3(9,3 \%)$ & $0(0 \%)$ & $1(4,5 \%)$ & $0(0 \%)$ \\
\hline ТИА & $1(3,1 \%)$ & $0(0 \%)$ & $0(0 \%)$ & $2(6,4 \%)$ \\
\hline Атеросклероз периферических артерий & $2(6,2 \%)$ & $1(16,6 \%)$ & $1(4,5 \%)$ & $3(9,6 \%)$ \\
\hline Гипергликемия в анамнезе & $3(9,3 \%)$ & $2(33,3 \%)$ & $4(18,1 \%)$ & $8(25,8 \%)$ \\
\hline
\end{tabular}

Примечание: *- наличие статистически достоверного различия, $p<0,05$. ИМТ — индекс массы тела; НГН — нарушенная гликемия натощак; НТГ — нарушенная толерантность к глюкозе; ОИМ — острый инфаркт миокарда; ОНМК — острое нарушение мозгового кровообращения; ОТ — объем талии; ПТТГ — пероральный тест толерантности к глюкозе; ТИА — транзиторная ишемическая атака; СД — сахарный диабет.

Таблица 2. Распределение пациентов в зависимости от степени нарушения углеводного обмена по результатам ПттГ

\begin{tabular}{|c|c|c|c|c|}
\hline \multirow{2}{*}{$\begin{array}{c}\text { Состояние углеводного } \\
\text { обмена }\end{array}$} & \multicolumn{2}{|c|}{$n=69$} & \multirow{2}{*}{ Х2/ $\varphi$} & \multirow{2}{*}{$p$} \\
\cline { 2 - 4 } & ГВПН 5,6-6,0 & ГВПН 6,1-6,9 & 6,5 & $0,011^{\star}$ \\
\hline Норма & $22(31,8 \%)$ & $10(14,4 \%)$ & 0,43 & $>0,05$ \\
\hline НГН & $2(2,8 \%)$ & $4(5,7 \%)$ & 1,9 & 0,17 \\
\hline НТГ & $8(11,5 \%)$ & $14(20,2 \%)$ & 0,3 & $>0,05$ \\
\hline СД & $3(4,3 \%)$ & $6(8,6 \%)$ & & \\
\hline
\end{tabular}

Примечание: *- наличие статистически достоверного различия, $p<0,05$. ГВПН - глюкоза венозной плазмы натощак; НГН - нарушенная гликемия натощак; НТГ — нарушенная толерантность к глюкозе; ПтТГ — пероральный тест толерантности к глюкозе; СД — сахарный диабет. В группе пациентов с исходным уровнем ГВПН 5,6-6,0 ммоль/л согласно результатам ПТТГ частота случаев с НГН составила 2,8\%, НТГ — 11,5\% и СД 2-го типа - 4,3\%. Таким образом, частота случаев возникновения предиабета составила 14,4\%. При сравнении итогов ПТТГ среди пациентов двух групп в зависимости от исходного уровня ГВПН достоверно меньше случаев с НТГ выявлено среди лиц с уровнем ГВПН 6,1-6,9 ммоль/л. 


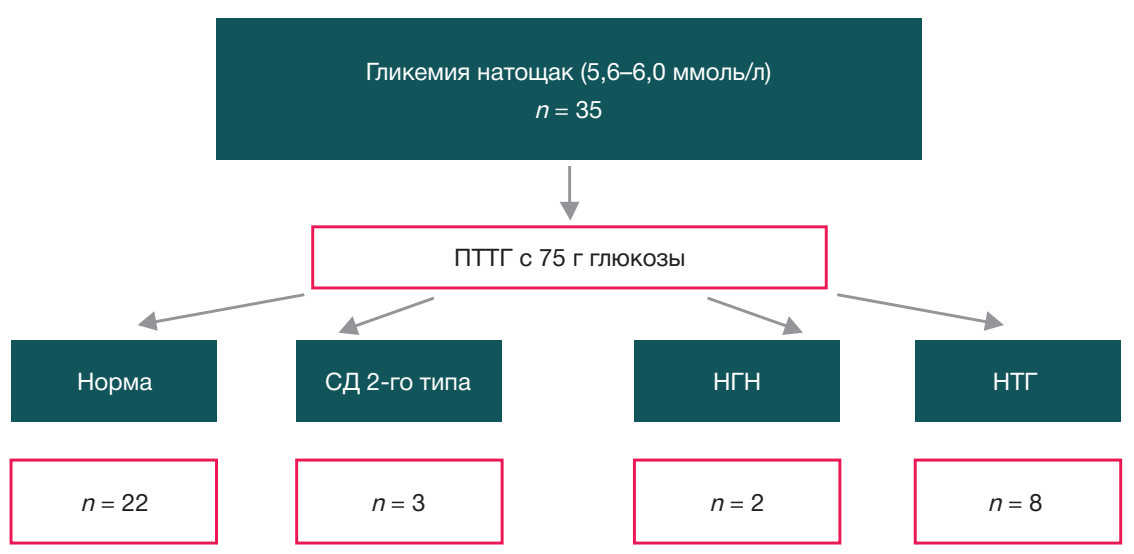

Рис. 3. Распределение пациентов в зависимости от степени нарушения углеводного обмена по результатам ПтТГ в группе из 35 человек с исходным уровнем ГВПН 5,6-6,0 ммоль/л. НГН — нарушенная гликемия натощак; НТГ — нарушенная толерантность к глюкозе; ПТТГ — пероральный тест толерантности к глюкозе

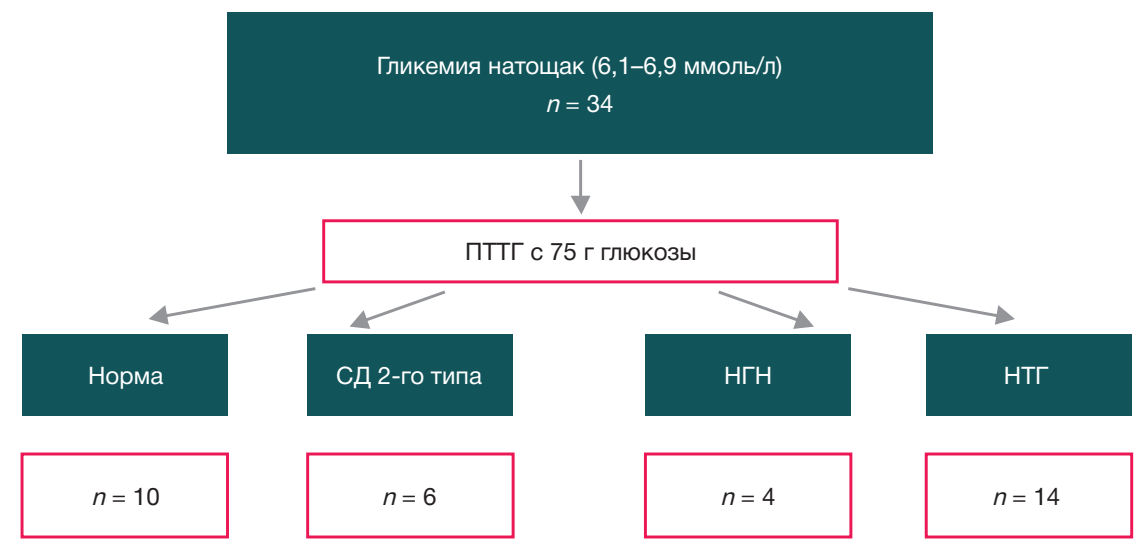

Рис. 4. Распределение пациентов в зависимости от степени нарушения углеводного обмена по результатам ПТТГ в группе из 34 человек с исходным уровнем ГВПН 6,1-6,9 ммоль/л. НГН — нарушенная гликемия натощак; НТГ- нарушенная толерантность к глюкозе; ПТТГ- пероральный тест толерантности к глюкозе

по данным лабораторных исследований, но по этому поводу к эндокринологу пациенты не обращались. ПТТГ был проведен во время госпитализации в дневной стационар по поводу дестабилизации значений АД.

Все пациенты с нарушениями углеводного обмена были осмотрены эндокринологом. Пациентам с впервые выявленным СД 2-го типа была назначена соответствующая сахароснижающая терапия.

Пациентам с НГН и НТГ с целью профилактики развития СД 2-го типа был назначен метформин в стартовой дозе 500 мг на ночь и даны рекомендации по самоконтролю уровня гликемии в домашних условиях. В случае нормогликемии было рекомендовано проведение контрольного ретеста через год, при повышении уровня гликемии - обращение к эндокринологу с целью коррекции медикаментозной терапии.

Лицам с нормальной толерантностью к глюкозе с учетом наличия факторов риска развития СД 2-го типа были даны рекомендации по правильному гипокалорийному питанию, снижению веса, приему гипотензивных препаратов при артериальной гипертензии и статинов в случае дислипидемии.

Таким образом, проведение ПТТГ у пациентов с уровнем ГВПН 5,6-6,0 ммоль/л, имеющих факторы риска развития СД и/или метаболический синдром, является актуальным.

\section{ОБСУЖДЕНИЕ РЕЗУЛЬТАТОВ}

В исследовании с участием 91 пациента дневного стационара с пограничным значением гликемии натощак и хотя бы одним фактором риска СД 2-го типа частота случаев впервые выявленного СД 2-го типа составила 34,0\% (n = 31), НГН $-6,5 \%(n=6)$, НТГ $-24,1 \%(n=22)$. Нормальная толерантность к глюкозе диагностирована в $35,1 \%(n=32)$ случаев.

Согласно исследованию NATION около 21 млн жителей РФ в возрасте 20-79 лет имеют предиабет, а еще 4,2 млн недиагностированный СД 2-го типа [3]. В данном исследовании в качестве диагностического критерия использовали анализ на гликированный гемоглобин. Такой подход является альтернативой [12] по отношению к использованному в данном исследовании, имеет свои преимущества (более высокую специфичность для выявления СД 2-го типа) и недостатки (более низкую специфичность для выявления предиабета) $[13,14]$.

Тот факт, что не менее 50\% пациентов с СД 2-го типа не знают о наличии у них заболевания, может свидетельствовать о недостаточной чувствительности принятых в РФ критериев отбора пациентов для скрининга. Определение группы риска СД 2-го типа в разных странах значительно отличается. В частности, Канадская диабетологическая ассоциация (CDA) указывает на необходимость проведения скрининга на СД 2-го типа у всех пациентов старше 40 лет вне зависимости от наличия у них факторов риска [15]. Американская диабетологическая ассоциация (ADA) определяет среди критериев для скрининга возраст старше 45 лет вне зависимости от наличия факторов риска, либо возраст моложе 45 лет + наличие определенных факторов риска [16]. Европейские клинические рекомендации предлагают использовать расчет риска СД 2-го типа по опроснику (калькулятору риска) и последующее 
проведение скрининга в популяциях среднего и высокого риска, исходя из результата заполнения опросника [17]. Столь существенное различие рекомендаций связано с экономическими и эпидемиологическими причинами.

Проведенное нами исследование показало необходимость расширения популяции скрининга в группах риска по развитию СД 2-го типа. Дано обоснование проведения ПТТГ с 75 г глюкозы всем лицам с уровнем ГВПН 5,6-6,0 ммоль/л, имеющим один или более фактор риска развития СД 2-го типа и/или метаболический синдром: среди данной категории СД был выявлен в 4,3\%, а предиабет - в 14,4\% случаев. Ранняя диагностика начальных нарушений углеводного обмена и своевременная медикаментозная профилактика позволят предотвратить конверсию предиабета в СД 2-го типа. Своевременное выявление СД 2-го типа и, соответственно, назначенное лечение помогут предупредить развитие осложнений.

\section{ВЫВОДЫ}

1) Проведенный скрининг состояния углеводного обмена у пациентов с пограничными значениями гликемии натощак в условиях дневного стационара продемонстрировал, что реальная распространенность СД 2-го типа в 3 раза превышает регистрируемую. 2) Необходимо проводить скрининг нарушений углеводного обмена среди пациентов групп риска по развитию СД 2-го типа с использованием анализа на ГВПН, в том числе при отсутствии у данных пациентов клинических проявлений диабета. 3) С целью уточнения состояния углеводного обмена проведение ПТТГ с 75 г глюкозы оправдано у всех лиц с метаболическим синдромом и/или наличием одного или более фактора риска развития СД 2-го типа, имеющих уровень ГВПН от 5,6 до 6,0 ммоль/л помимо традиционного показания лицам с уровнем ГВПН от 6,1 до 6,9 ммоль/л.

\section{Литература}

1. International Diabetes Federation. Diabetes Atlas, $8^{\text {th }}$ ed, 2017.

2. Государственный регистр сахарного диабета. Профессиональный всероссийский ресурс по нозологиям диабета под эгидой Эндокринологического Научного Центра. Доступно по ссылке: http://diaregistry.ru/content/o-proekte.html\#content. Проверено 28.11.18.

3. Дедов И. И., Шестакова М. В., редакторы. Алгоритмы специализированной медицинской помощи больным сахарным диабетом. 8-е издание. М., 2017.

4. Дедов И. И., Шестакова М. В., Галстян Г. Р. Распространенность сахарного диабета 2 типа у взрослого населения России (исследование NATION). Сахарный диабет. 2016; 19 (2): 104-12.

5. Результаты реализации подпрограммы «Сахарный диабет» Федеральной целевой программы «Предупреждение и борьба с социально значимыми заболеваниями 2007-2012 гг». Под ред. Дедова И. И., Шестаковой М. В. Сахарный диабет. 2013 Спецвыпуск 2S: 2-48.

6. Тамбовское областное государственное бюджетное учреждение «Центр материально-технического обеспечения деятельности учреждений здравоохранения». Форма №12. Приказ Росстата: Об утверждении формы от 21.07.2016; № 355.

7. Стародубова А. В., Червякова Ю. В., Копелев А. А., Алиева А. М. Возможности медикаментозной коррекции метаболических нарушений и профилактики сахарного диабета при нарушениях углеводного обмена. Лечебное дело. 2015; (3): 59-65.

8. van Vliet-Ostaptchouk J, Nuotio M, Slagter S, Doiron D, Fischer K, Foco $L$, et al. The prevalence of metabolic syndrome and metabolically healthy obesity in Europe: a collaborative analysis of ten large cohort studies. BMC Endocrine Disorders. 2014; 14 (1), 9.

9. Симоненко В. Б., Медведев И. М., Толмачев В. В.

Патогенетические аспекты артериальной гипертензии при метаболическом синдроме. Клиническая медицина. 2011; 89 (1): 49-51.

10. Ивашкин В. Т., Драпкина О. М., Корнеева О. Н. Клинические варианты метаболического синдрома. М.: Медицинское информационное агентство, 2011; с. 220.

11. Международная Федерация диабета (IDF): консенсус по критериям метаболического синдрома. Ожирение и метаболизм. 2005; (3): 47-9.

12. Khetan A, Rajagopalan S, Prediabetes. Can J Cardiol. 2018 May; 34 (5): 615-23.

13. Yan S. et al. Diagnostic accuracy of HbA1c in diabetes between Eastern and Western. Eur J Clin Invest. 2013 Jul; 43 (7): 716-26.

14. Barry E. Efficacy and effectiveness of screen and treat policies in prevention of type 2 diabetes: systematic review and metaanalysis of screening tests and interventions. BMJ. 2017 Jan; 4; 356: 16538.

15. Goldenberg R, Punthakee Z. Canadian Diabetes Association 2013 clinical practice guidelines for the prevention and management of diabetes in Canada: definition, classification and diagnosis of diabetes, prediabetes and metabolic syndrome. Can J Diabetes. 2013; 37.

16. Classification and diagnosis of diabetes. Diabetes Care 2017; (40): 11-24

17. ESC Guidelines on diabetes, pre-diabetes, and cardiovascular diseases developed in collaboration with the EASD: the task force on diabetes, pre-diabetes, and cardiovascular diseases of the European Society Of Cardiology (ESC) and Developed in Collaboration With The European Association For The Study Of Diabetes (EASD). Eur Heart J. 2013; (34): 3035-87.

\section{References}

1. International Diabetes Federation. Diabetes Atlas, $8^{\text {th }}$ ed, 2017.

2. State Diabetes Register. Professional All-Russian Resource on Diabetes Nosology under the auspices of Endocrinology Research Center/ http://diaregistry.ru/content/o-proekte.html\#content Verified on May 28, 2018.

3. Dedov II, Shestakova MV, editors. Algorithms of Specialized Medical Care for Diabetes Mellitus Patients. 8-th Edition. Moscow, 2017.

4. Dedov II, Shestakova MV, Galstyan GR. Prevalence of Type 2 Diabetes Mellitus in Adult Russian Population (NATION study).

Diabetes Mellitus. 2016; 19 (2): 104-112.

5. Results of implementation of the subprogramme «Diabetes Mellitus» of the Federal Target Program «Prevention and Control of Socially Significant Diseases in 2007-2012». Edited by Dedov II., Shestakova MV. Diabetes Mellitus. 2013; Special edition 2S: 2-48.

6. Tambov Regional State Budgetary Establishment «Technical and Material Support Center of Healthcare Institutions Activities». Form No. 12. Order of the Federal State Statistics Service: On form approval dated 2016 July 21; 355.

7. Starodubova AV., Chervyakova YuV., Kopelev AA., Alieva AM. 
Possibilities of Drug Correction of Metabolic Disorders and Prevention of Diabetes Mellitus in Case of Carbohydrate Metabolism Disorder. General Medicine. 2015; (3): 59-65.

8. van Vliet-Ostaptchouk J, Nuotio M, Slagter S, Doiron D, Fischer K, Foco L, et al. The prevalence of metabolic syndrome and metabolically healthy obesity in Europe: a collaborative analysis of ten large cohort studies. BMC Endocrine Disorders. 2014; 14 (1): 9.

9. Simonenko VB, Medvedev IM, Tolmachev W. Patogeneticheskie aspekty arterial'noj gipertenzii pri metabolicheskom sindrome/ Klinich. medicina. 2011; 89 (1): 49-51.

10. Ivashkin VT, Drapkina OM, Korneeva ON. Klinicheskie varianty metabolicheskogo sindroma. M: Medicinskoe informacionnoe agentstvo. 2011; 220.

11. International Diabetes Federation (IDF): Consensus on the Criteria of Metabolic Syndrome. Obesity and Metabolism. 2005; (3): 47-9.

12. Khetan A, Rajagopalan S, Prediabetes, Can J Cardiol. 2018 May; 34(5): 615-23

13. Yan $\mathrm{S}$, et al. Diagnostic accuracy of $\mathrm{HbA} 1 \mathrm{c}$ in diabetes between
Eastern and Western. Eur J Clin Invest. 2013 Jul; 43(7): 716-26.

14. Barry E. Efficacy and effectiveness of screen and treat policies in prevention of type 2 diabetes: systematic review and metaanalysis of screening tests and interventions. BMJ. 2017 Jan; 4; 356: i6538.

15. Goldenberg R, Punthakee Z. Canadian Diabetes Association 2013 clinical practice guidelines for the prevention and management of diabetes in Canada: definition, classification and diagnosis of diabetes, prediabetes and metabolic syndrome. Can J Diabetes. 2013; 37.

16. Classification and diagnosis of diabetes. Diabetes Care. 2017; (40): 11-24

17. ESC Guidelines on diabetes, pre-diabetes, and cardiovascular diseases developed in collaboration with the EASD: the task force on diabetes, pre-diabetes, and cardiovascular diseases of the European Society Of Cardiology (ESC) and Developed in Collaboration With The European Association For The Study Of Diabetes (EASD). Eur Heart J. 2013; (34): 3035-87. 